\title{
Data pre-processing using an FPGA by binning gamma ray energies and forwarding consolidated spectra data
}

\author{
Ana Fernandes ${ }^{a}$,, Rita C. Pereira ${ }^{a}$, André C. Neto ${ }^{b}$, Jorge Sousa ${ }^{a}$, Bernardo B. Carvalho ${ }^{a}$, \\ Vasily Kiptily $^{\mathrm{c}}$, Carlos M.B.A. Correia ${ }^{\mathrm{d}}$, Bruno Gonçalves ${ }^{\mathrm{a}}$, JET EFDA Contributors ${ }^{1}$ \\ a Instituto de Plasmas e Fusão Nuclear, Instituto Superior Técnico, Universidade de Lisboa, 1049-001 Lisboa, Portugal \\ ${ }^{\mathrm{b}}$ Fusion for Energy, 08019 Barcelona, Spain \\ c CCFE, Culham Science Centre, Abingdon OX14 3DB, UK \\ d Centro de Instrumentação, Dept. de Física, Universidade de Coimbra, 3004-516 Coimbra, Portugal
}

\section{H I G H L I G H T S}

- JET monitor equipped with a DAQ system to digitize gamma signals from 19 detectors.

- DAQ FPGAs used for real-time processing and streaming the gamma-ray energy values.

- New algorithm was designed to produce at FPGA real-time spectra from energy values.

- Spectra built at FPGA ensures streamed packets not limited by count rate.

- New algorithm is relevant for future experiments expecting high event count rates.

\section{A R T I C L E I N F O}

\section{Article history:}

Received 19 September 2014

Received in revised form 5 June 2015

Accepted 8 June 2015

Available online 2 July 2015

\section{Keywords:}

FPGA

Digital pulse processing

Real-time processing

Spectroscopy

\begin{abstract}
A B S T R A C T
Gamma-ray diagnostics are considered of crucial importance for understanding the plasma behavior of next fusion devices. Among other physical phenomena, gamma-ray spectra can provide information about the fusion reactions rate and the fast ions temperature and confinement, indicators of how close we are from reaching self-sustained burning plasmas. Accordingly, dedicated gamma-ray diagnostics are currently installed at the Joint European Torus (JET). The 2D gamma-ray profile monitor is one of these diagnostics, equipped with an Advanced Telecommunications Computing Architecture (ATCA) Data Acquisition (DAQ) system, capable of digitizing gamma-ray signals from the 19 photodiode detectors. The DAQ system includes Field Programmable Gate Array (FPGA) devices, with embedded processing algorithms. These algorithms are responsible for processing the gamma-ray signals acquired from each detector in real-time, and for periodically streaming the corresponding energy values to the DAQ host. However, for higher count rates it will be unfeasible to stream periodically all the energy values without loss. Thus, a new algorithm was designed, capable to produce real-time spectra at FPGA from the processed energy values. The spectra should be periodically streamed, delivering binned data rather than a value for each gamma-ray. This allows to reduce the data rate, avoiding data losses. Consequently, the streaming data can be used for control purposes, as demanded by next fusion experiments with long plasma discharges of high energy/count rate content. This work describes the real-time spectrum code developed for FPGA along with the attained results. It was concluded that the spectrum code is suitable for implementation in any spectroscopy diagnostic, whenever real-time spectra are required.
\end{abstract}

(C) 2015 EURATOM. Published by Elsevier B.V. All rights reserved.

\footnotetext{
* Corresponding author.

E-mail address: anaf@ipfn.ist.utl.pt (A. Fernandes).

${ }^{1}$ See the Appendix of F. Romanelli et al., Proceedings of the 24th IAEA Fusion Energy Conference 2012, San Diego, USA.
}

\section{Introduction}

Confined alpha particles produced from Deuterium-$\operatorname{Tritium}(D T)$ reactions should be responsible for the self sustained plasma heating of future fusion reactors [1]. It is expected that these alpha particles transfer their energy to the thermal plasma providing the necessary heating for a steady state operation [2]. Thus, 
understanding alpha particles behavior during DT experiments is of crucial importance to reach self sustained plasmas [3].

It is known that intense gamma-ray emissions can be measured during interactions between alpha particles (or energetic ions) and impurities, providing information about the fusion reactions rate [4]. Accordingly, gamma-ray spectroscopy is one of the proposed techniques for the measurement of alpha particles and fast ions ( $\mathrm{MeV}$ range) populations in future plasmas. Furthermore, it is known that alpha particles and fast ions may drive plasma instabilities, potentially damaging its confinement [2], [5]. Therefore, real-time measurements of gamma-ray emission will be required in future experiments for proper plasma control and confinement.

Several gamma-ray diagnostics were installed at JET, the only present machine capable to confine fast ions ( $\mathrm{MeV}$ range) produced by external sources (ICRF and NBI auxiliary heating sources) [6]. One of the diagnostics is the JET 2D gamma-ray profile monitor, composed by 19 lines-of-sight to view the plasma through $\mathrm{CsI}(\mathrm{Tl})$ detectors. The diagnostic was recently upgraded with an Advanced Telecommunications Computing Architecture (ATCA) Data Acquisition (DAQ) system developed by Instituto superior Técnico (IST). ATCA technology has been extensively used by several fusion diagnostics, being considered for ITER diagnostics targeting the plasma control [7]. The DAQ system of the JET gamma-ray profile monitor includes real-time processing and data transfer capabilities, allowing to process and stream periodically, during discharges, packets with the gamma-ray energy values [8].

However, streaming all gamma-ray energy values requires count rate dependent packet sizes, which is not the optimal approach. Moreover, for high count rates it may be unfeasible streaming all energy values without losses. Accordingly, a new FPGA algorithm was designed capable to deliver the energy spectra from the processed energy values. Thereby, the new streaming packet, with standardized size, should publish the energy spectra (binned data) rather than an energy value for each gamma-ray. This new algorithm will be relevant for future $D T$ experiments where much higher count rates are expected. So far, the poor SNR and the $\mathrm{CsI}(\mathrm{Tl})$ detectors slow response limit the diagnostic performance to a maximum count rate of $20 \mathrm{k}$ events/s. Nevertheless, this limitation will be overcome with an expected set of new detectors.

Thus, Section 2 describes the real-time pulse processing and data transfer architecture of the JET gamma-ray profile monitor, Section 3 details the new FPGA real-time spectrum code and Section 4 presents the laboratory results.

\section{The real-time system}

The DAQ system of the JET gamma ray profile monitor is composed by an ATCA shelf with 3 digitizer modules connected to a controller [9] through PCI-express (PCIe) links. Each digitizer module [10] includes eight 13-bit resolution Analog to Digital Converters (ADCs) with a maximum sampling rate of 250 M Samples/s, and two Field Programmable Gate Array (FPGA) devices (XC4VFX60). Besides the basic module functionality, FPGAs are used for real-time processing, delivering the energy values of acquired pulses. The system is also connected to the JET real-time network through an Asynchronous Transfer Mode (ATM) module installed in the ATCA controller. The ATM module is responsible for publishing during discharges packets with counts from established energy windows.

The interface to the JET Control and Data Acquisition System (CODAS), and to the JET real-time data network, is provided by the Multithreaded Application Real-Time executor (MARTe), the C++ framework installed in the ATCA controller under Linux OS with kernel version 2.6.35.9.

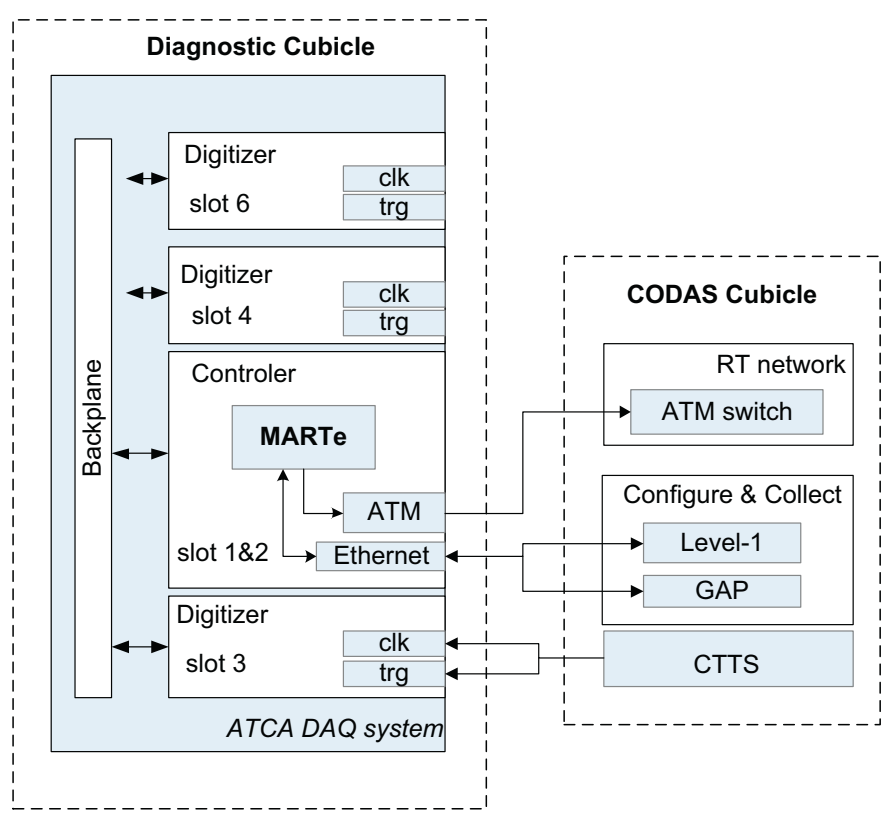

Fig. 1. Schematic representation of the DAQ system and its interface with JET CODAS sub-systems (diagnostic configuration (Level-1), data collection (GAP), ATM network, JET absolute time (CTTS).

In Fig. 1 is depicted a schematic representation of the DAQ system and its interface to JET CODAS sub-systems.

As described in Fig. 2, for each JET shot the acquisition is enabled and the FPGAs start to process the incoming gamma-ray signals, calculating the corresponding energy values. Simultaneously, packets with processed data are streamed from the digitizers to the ATCA controller. During acquisition, a total of five real-time packets with processed data are streamed, precisely at every $2 \mathrm{~ms}$, covering all the $19 \mathrm{CsI}(\mathrm{Tl})$ signals. The total number of energy values per channel, that can be transferred in each packet, is $n=247$. This value results from a compromise between FPGA resources and the $1 \mathrm{M} \mathrm{Hz}$ JET clock used to build those packets. Nevertheless, considering the maximum count rate of $20 \mathrm{kevents} / \mathrm{s}$ attained by the $\mathrm{CsI}(\mathrm{Tl})$ detectors, it is possible to conclude that the packets size still oversized for this diagnostic.

The packets synchronization is ensured by JET absolute time signals (clock and trigger) connected to the master FPGA. A dedicated software code running in the MARTe application is responsible for building calibrated spectra from the streamed energy values, and determining from each spectra the total counts in predefined energy windows.

However, for higher count rates, the maximum number of energy values may overflow the packets size (e.g. $1 \mathrm{M}$ events/s are expected during DT experiments, producing up to $8 \mathrm{k}$ energy values in each $2 \mathrm{~ms}$, per packet - 4 channels).

Thus, a new code was developed capable to cope with the count rate independent packets goal, as detailed in Section 3.

\section{The real-time spectrum code}

The developed real-time spectrum algorithm is capable of producing real-time spectra at FPGA from the processed energy values. As depicted in Fig. 3 diagram, each time an energy value is found the algorithm starts to determine the corresponding energy bin. Each energy bin defines a different address in the FPGA spectrum buffer (SPECTRUM). Thereafter, the SPECTRUM address found is incremented by one (SPECTRUM[addr]++).

The total number of SPECTRUM addresses (energy bins) is 256 , and the maximum number of counts (energy values) per 

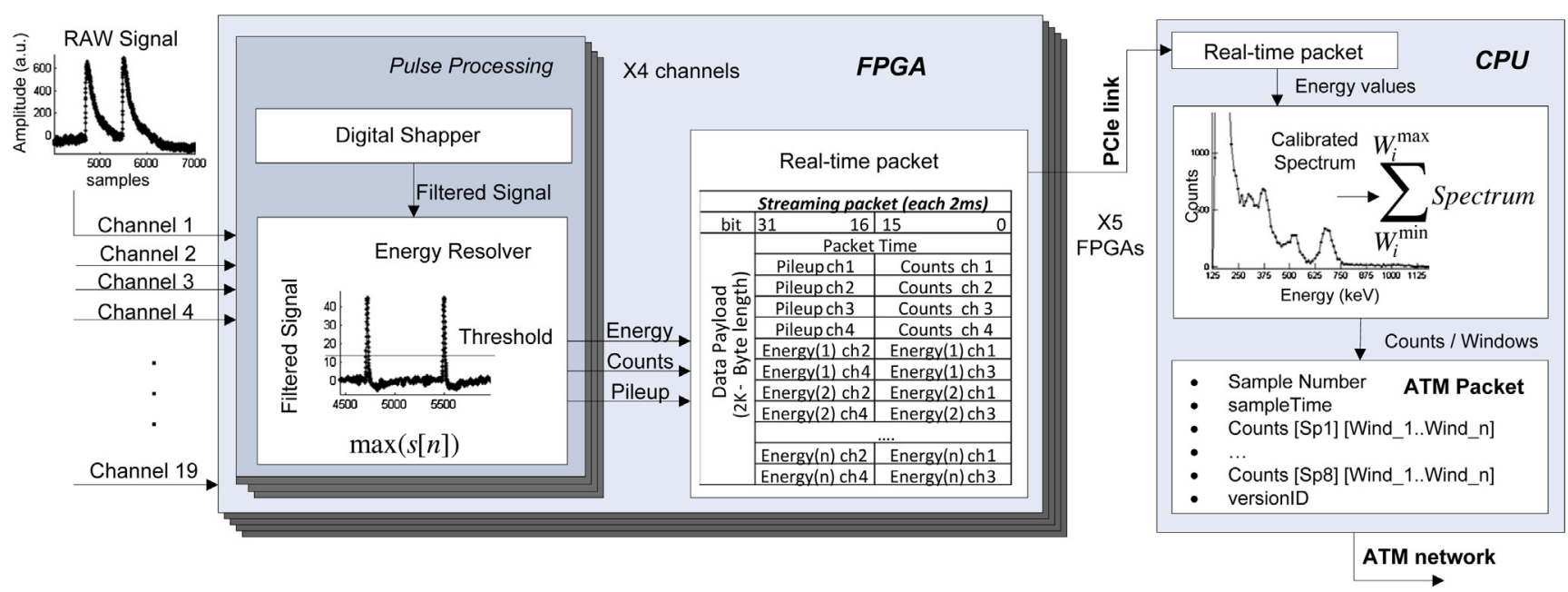

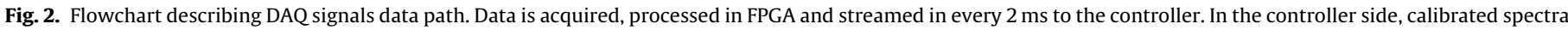
are produced with streamed data, and counts from interesting energy windows are delivered to the real-time network.

SPECTRUM address is $65 \mathrm{~K}$ (16-bit width). Periodically (each $2 \mathrm{~ms}$ ), the SPECTRUM buffer is copied to an equal size buffer (SP) for serial streaming. When all SP buffer addresses were streamed, is made a new SPECTRUM buffer copy (SP = SPECTRUM), the SPECTRUM data is deleted, and the process repeats.

Considering the FPGA limitations when operating with floating points, the streamed spectra are not calibrated. Therefore, host is responsible for converting the spectra bins in energies range from estimated calibration slopes.

Depending on the required energy resolution, the energy bins window may need to be adjusted. This can be done through a compromise between energy windows and total energy range, without changing the packets size. As example, if higher spectra resolution is required, the energy bins window should be reduced, as well as the total energy SPECTRUM range, keeping the total number of SPECTRUM addresses.

\section{Results}

The developed spectrum code was tested in laboratory. For that purpose it was used the KC705 developing kit from Xilinx with an embedded Kintex-7 FPGA. The KC705 board is connected through an $\mathrm{x} 4$ PCle link (GEN 1, 2 GB/s of bandwidth) to the host PC. Two different experimental setups were used to simulate data from ADCs.
The first setup uses energy values stored in an FPGA embedded block of memory (BRAM). Those energy values were obtained by the profile monitor DAQ system, in presence of ${ }^{22} \mathrm{Na}$ and ${ }^{137} \mathrm{Cs}$ radioactive sources. The ${ }^{22} \mathrm{Na}$ source, embedded in the detectors set, emit two gamma-ray peaks at $511 \mathrm{keV}$ and $1275 \mathrm{keV}$, and the ${ }^{137} \mathrm{Cs}$ source, embedded in collimators near detectors, emit a $662 \mathrm{keV}$ peak. During operation, the FPGA code receives the energy values stored in BRAM and finds the corresponding energy bin in the SPECTRUM addresses. The resulting spectrum is periodically streamed (each $2 \mathrm{~ms}$ ) to the host PC, as described in Section 3. In the resulting spectrum it is possible to observe the expected energy peaks, as depicted in Fig. 4.

Moreover, it is possible to conclude that real-time spectra are similar to results obtained by offline analysis, detailed in Section 2 . It is important to remark that limited detection efficiencies are expected from gamma-ray profile monitor. The spectral lines resolution is affected by the tokamak geometry, uncertainty due to the nonlinear response of $\mathrm{CsI}(\mathrm{Tl})$ detectors, the absorber materials in each Line-Of-Sight (LOS), and low counting statistics [11,12].

The second setup uses synthetic data from FPGA. Accordingly, 3 different values, corresponding to 3 distinct energy peaks, were periodically delivered to the real-time spectrum algorithm. Those peaks were defined according with the following frequency relation: $f_{3}=1 / 3 f_{2}=2 / 3 f_{1}$, where $f_{1}=100 \mathrm{kHz}$.

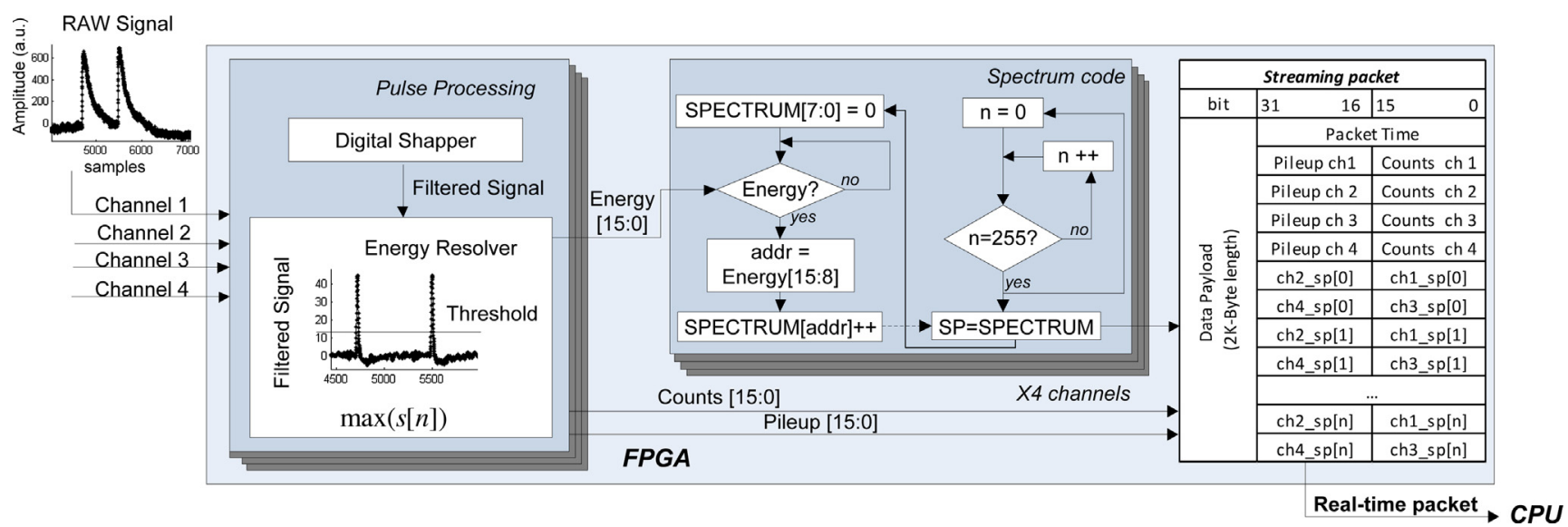

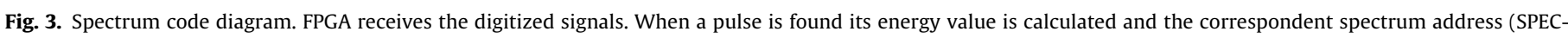
TRUM[addr]) is incremented by one. Periodically (when $n=255$ ) the produced spectrum is copied to an equal buffer (SP) which is streamed from FPGA to host. 


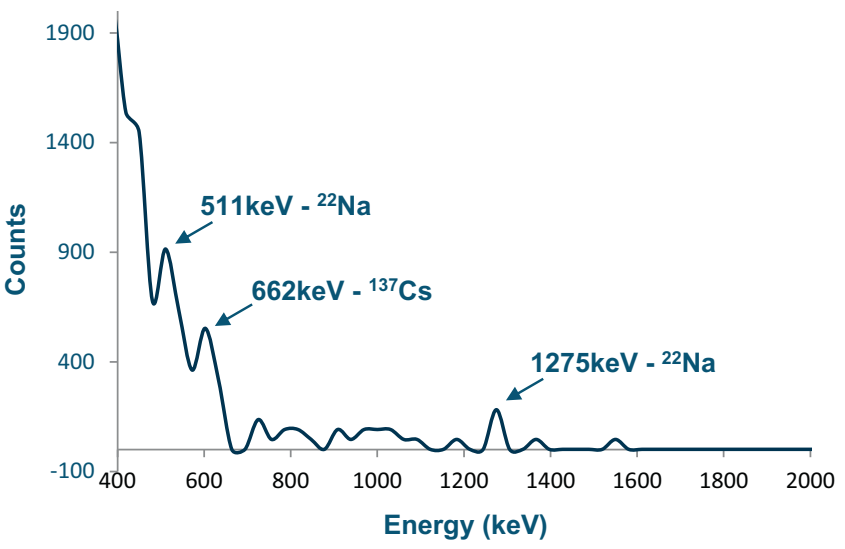

Fig. 4. Real-time spectrum from KC705 FPGA processing code. Energy values acquired with the profile monitor in presence of ${ }^{22} \mathrm{Na}$ and ${ }^{137} \mathrm{Cs}$ radioactive sources were used as input data. The expected 511, 662 and $1275 \mathrm{keV}$ are visible.

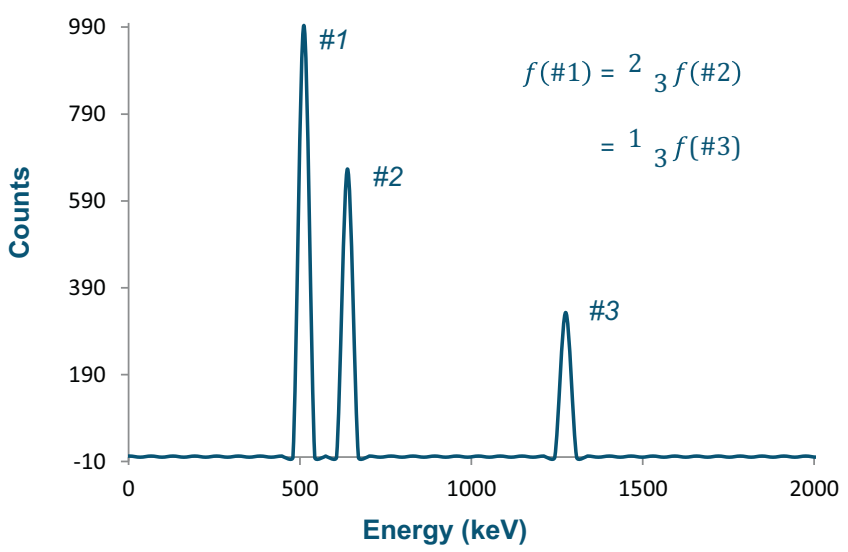

Fig. 5. Spectrum from FPGA synthetic data. 3 different peaks were defined according with the following frequency relation: $f_{3}=1 / 3 f_{2}=2 / 3 f_{1}$.

As depicted in Fig. 5, the corresponding real-time spectra agree with the expected results.

An offline software code was used to calibrate real-time spectra of both setups.

\section{Conclusions}

The new FPGA spectrum code is capable to determine the energy spectra from energy values in real-time. Thus, the algorithm allows to have count rate independent packets ensuring no data losses. Moreover, it is possible to change the spectra resolution, by reordering the streaming packets structure without changing its size.

This spectrum code is relevant for future DT experiments at JET, as well as for future fusion diagnostics expecting high event count rates. Furthermore it is suitable for implementation in other spectroscopy applications whenever real-time spectra are required.

The code was tested in the KC705 development kit from Xilinx. The kit FPGA is different from the JET DAQ system FPGAs. As so, the code performance must also be tested on-site. Nevertheless, the KC705 Kintex FPGA is fully compatible with the new ultra fast ATCA Advanced Mezzanine Card (AMC) FPGA developed by IST. This AMC was specially conceived for real-time spectroscopy applications expecting high event count rates [13].

\section{Acknowledgements}

This work was supported by EURATOM and carried out within the framework of the European Fusion Development Agreement. This work has been carried out within the framework of the EUROfusion Consortium and has received funding from the European Union's Horizon 2020 research and innovation programme under grant agreement number 633053. The views and opinions expressed herein do not necessarily reflect those of the European Commission. Moreover, Fusion for Energy cannot be held responsible for any use which may be made of the information contained therein. IST activities also received financial support from "Fundação para a Ciência e Tecnologia" through project PestOE/SADG/LA0010/2013.

\section{References}

[1] M. Sasao, T. Nishitani, A. Krasilnilov, S. Popovishev, V. Kiptily, J. Kallne, Fusion product diagnostics, Fusion Sci. Technol. 8 (2) (2008) 604-639.

[2] P.R. Thomas, et al., Observation of alpha heating in jet DT plasmas, Phys. Rev. Lett. 80 (1998) 5548-5551.

[3] V. Kiptily, et al., Fusion alpha-particle diagnostics for DT experiments on the joint European torus, in: Proceedings of the International Conference on Fusion Reactor Diagnostics, Villa Monastero, Varenna, Italy, 2013, xx.

[4] V.G. Kiptily, S. Popovichev, S.E. Sharapov, L. Bertalot, F.E. Cecil, S. Conroy, M.J. Mantsinen, Gamma-diagnostics of alpha-particles in ${ }^{4} \mathrm{He}$ and D-T plasmas, Rev. Sci. Instrum. 74 (3) (2003) 1753-1756.

[5] K.M. Young, Plasma measurements: an overview of requirements and status, Fusion Sci. Technol. 53 (2008) 281-303.

[6] M. Nocente, et al., High-resolution gamma ray spectroscopy measurements of the fast ion energy distribution in JET ${ }^{4}$ He plasmas, Nuclear Fusion 52 (6) (2012) 063009

[7] B. Gonçalves, J. Sousa, B. Carvalho, A. Batista, A. Neto, B. Santos, A. Duarte, D. Valcárcel, D. Alves, M. Correia, A. Rodrigues, P. Carvalho, M. Ruiz, J. Vega, R. Castro, J. Lopez, N. Utzel, P. Makijarvi, ITER fast plant system controller prototype based on ATCA platform, Fusion Eng. Des. 87 (12) (2012) 2024-2029.

[8] A.M. Fernandes, R.C. Pereira, A. Neto, D.F. Valcárcel, D. Alves, J. Sousa, B.B. Carvalho, V. Kiptily, B. Syme, P. Blanchard, A. Murari, C.M.B.A. Correia, C.A.F. Varandas, Real-time processing system for the JET hard X-ray and gamma-ray profile monitor enhancement, IEEE Trans. Nuclear Sci. 61 (3) (2014) 1209-1215.

[9] A. Batista, A. Neto, M. Correia, A. Fernandes, B. Carvalho, J. Fortunato, J. Sousa, C. Varandas, F. Sartori, M. Jennison, ATCA control system hardware for the plasma vertical stabilization in the JET tokamak, IEEE Trans. Nuclear Sci. 7 (2) (2010) 583-588.

[10] R. Pereira, J. Sousa, A. Fernandes, F. Patrício, B. Carvalho, A. Neto, C. Varandas, G. Gorini, M. Tardocchi, D. Gin, A. Shevelev, ATCA data acquisition system for gamma-ray spectrometry, Fusion Eng. Des. 83 (23) (2008) 341-345.

[11] B. Esposito, R.M. Solis, P. van Belle, O.N. Jarvis, F.B. Marcus, G. Sadler, R. Sanchez, B. Fischer, P. Froissard, J.M. Adams, E. Cecil, N. Watkins, Runaway electron measurements in the JET tokamak, Plasma Phys. Control. Fusion 38 (12) (1996) 2035

[12] C. Schlatter, Gamma Ray Spectrometry in Tokamaks, CRPP Internal Report Int 212/06, CRPP, 2006.

[13] R. Pereira, A. Combo, M. Correia, A. Rodrigues, A. Fernandes, J. Sousa, C Correia, B. Gonçalves, C. Varandas, Ultra high-frequency data acquisition AMC module for high performance applications, Fusion Eng. Des. 88 (6-8) (2013) 1409-1413. 